\title{
Reappraisal of the Mechanisms of Glaucomatous Optic Nerve Damage
}

\author{
HARRY A. QUIGLEY \\ Baltimore, Maryland
}

Glaucoma is a progressive optic neuropathy with a typical optic disc appearance and a particular loss of sensitivity in visual field testing. Its disc appearance includes enlargement of the disc cup toward the rim, undercutting of the rim tissue, and deepening of the cup floor. These features are all included in the term glaucomatous cupping, though the individual nerve head may exhibit only some of them. Some eyes can simulate glaucoma cupping even though they are normal or have another optic neuropathy, but these are rare. ${ }^{1}$ In glaucoma, this pattern is recognised in nearly every case. In a visual field test, glaucoma first shows abnormality between 5 and 30 degrees from fixation. The abnormality expands into an arcuate shape, isolating the central and temporal field into islands. Again, while other disorders can simulate this field pattern, they almost never have glaucomatous cupping.

Most glaucoma eyes have an intraocular pressure (IOP) that is higher and more unstable than normal. However, all eyes with elevated IOP do not have glaucomatous optic neuropathy at the time abnormal IOP is discovered. This does not mean that these eyes do not have glaucoma or that they never will have it. Some have not had elevated IOP long enough to be damaged. Others are already damaged, but the visual field test is too insensitive to detect it. ${ }^{2}$ It is therefore deceptive to define glaucoma solely by field test results or to use a different diagnostic term (ocular hypertension) for persons with normal fields but elevated IOP.

Some have suggested that glaucomatous optic neuropathy frequently occurs without any past IOP elevation and that the two are not causally related. Our own study (the Baltimore Eye Survey) shows that most persons with glaucomatous neuropathy selected at random from the population have easily demonstrable elevated IOP. Only a tiny minority appear to have glaucoma damage and normal IOP (socalled low tension glaucoma). Glaucomatous neuropathy results from IOP elevation after steroid use, trauma, or inflammation. Chronic IOP elevation in a monkey eye leads to disc and nerve damage indistinguishable from that of the human eye. ${ }^{3}$ This brief report will summarise the recent additions to information about how elevated IOP causes optic nerve damage.

\section{Where Does Glaucoma Damage Nerve Fibres?}

Over the last 10 years, I examined more than 150 human glaucoma eyes with known clinical histories. ${ }^{3.4}$ This material indicated that the nerve fibre injury occured within the optic nerve head at the level of the sclera, the scleral lamina cribrosa. Here, axons were swollen with accumulations of their intracellular contents that would normally have been moving past this area, but were held up by the injury. Vrabec $^{5}$ confirmed that this is the location of injury in human glaucoma eyes, and it is the site of damage in chronic IOP elevation in monkeys, as well. The intracellular contents that accumulate at the scleral lamina in glaucoma move by axonal transport, the system by which the cell body supplies its axon

Professor of Ophthalmology and Director, Glaucoma Service, Wilmer Institute, Johns Hopkins University School of Medicine, Baltimore, Maryland, USA.

Correspondence to: Professor A. Quigley MD, Maumenee B-110, Wilmer, Johns Hopkins Hospital, Baltimore, MD 21205, USA. 
and synaptic terminals with materials. Interference with axonal transport is sufficient to cause cell death. But, axonal transport might be disturbed by anoxia, by mechanical compression, or by build-up of toxic substances. Hence, the fact that transport blockade is present in glaucomatous neuropathy does not establish what is (are) the intervening link(s) between elevated IOP and cell death.

\section{Optic Nerve Head Anatomy Explains Field Loss in Glaucoma}

The scleral lamina cribrosa consists of ten successive connective tissue plates with perforations through which neural bundles pass. Astrocytic glial cells surround the bundles, maintaining proper ionic concentration for neural impulse transmission. Optic nerve cross-sections from human glaucomatous eyes have a selectively greater loss of fibres entering the upper and lower optic disc poles. It is believed that these are the fibres that subserve the arcuate area in which typical glaucoma field loss occurs. The susceptible regions form the shape of an hourglass. Kirsch and Anderson ${ }^{6}$ observed that some glaucomatous eyes lose the upper or lower disc rim first, producing vertically oval cup shape or a local notch of rim loss at 6 or 120 'clock. Since the site of damage is the scleral lamina, and the pattern of loss is hourglass-shaped, I examined the laminar anatomy for some feature that would fit the damage pattern. ${ }^{7}$ With the scanning electron microscope, the upper and lower poles of the normal lamina cribrosa can be seen to have larger pores for the passage of nerve bundles. Hence, the structural support is less there than in the nasal and temporal nerve heads. The conclusion from these observations has direct implications for glaucoma pathogenesis. If IOP increases, two forces act on the lamina. Firstly, there is an inside-out push, tending to compress the laminar sheets and to bow them outward. Secondly, increased IOP raises scleral tension, pulling on the perimeter of the laminar sheets. Both actions might distort the lamina, pressing on either the nerve fibres themselves or on their nutrient capillaries that are located within the laminar sheets. Resistance to distortion would vary within the nerve head. The upper and lower poles, with the least collagenous tissue, would distort first, leading to greater damage to the bundles passing through their zones. Therefore, the pattern of hourglass optic atrophy is explained by the regional laminar architecture.

This hypothesis is local and is supported by examinations of human glaucomatous eyes. ${ }^{8}$ Compression of the lamina cribrosa is seen even in eyes that have not yet developed field loss. Laminar compression worsens with each succeeding stage of optic nerve damage. Most importantly, this change is greater at the superior and inferior poles than in the temporal-nasal axis.

\section{Does Loss of Glial Cells Contribute to Glaucoma Cupping?}

Seventy years ago, Fuchs reported that glaucomatous eyes lose glial cells of the optic nerve head. Since he had no accurate tonometry, gonioscopy, or field testing, we have no way of knowing how the eyes he studied would be classified today. Shaffer later suggested that loss of glial cells might cause the increased cup size that precedes field loss. In both human and experimental primate glaucomatous eyes, I have found no disproportionate loss of astrocytes (glial cells). ${ }^{4}$ Why, then, did Fuchs find that glia had disappeared? Astrocytes make up about $10 \%$ of the nerve head tissue. Once all nerve fibres are gone, the nerve head would look empty, even if all the glia were preserved. In nerve heads with advanced glaucoma damage, the lamina is compressed and expanded outward into the choroid and sclera. The surface area of the nerve head becomes 3 times greater than normal. The remaining glia are therefore spread out over a much bigger area and look fewer in number even if the original number is present.

\section{Further Steps in the Damage Pathway Direct mechanical effects on nerve fibres}

Might distortion and compression of the lamina cribrosa directly compress optic nerve fibres, leading to subsequent cell.death? Or, does laminar compression impair function of optic nerve head capillaries? The physical forces generated at the lamina could be considerable. Forces generated by laminar shape change could far exceed the simple increase in pressure gradient across the nerve head caused by elevated IOP. The normal gradient is IOP 
minus optic nerve tissue pressure, or about $10 \mathrm{~mm} \mathrm{Hg}$. With chronic glaucoma, this would increase to $20 \mathrm{~mm} \mathrm{Hg}$ at $\mathrm{IOP}=30 \mathrm{~mm} \mathrm{Hg}$. But, the shearing force caused by pinching of two adjacent laminar sheets could exceed this considerably. At these IOP levels, axonal transport is blocked under experimental conditions.

\section{Is vascular insufficiency a cause of neural loss?}

The evidence for vascular insufficiency as a cause for glaucomatous optic neuropathy is indirect. Previous reports discuss 'ischaemia' without defining what is meant by the term. In the central nervous system, fibre tracts supply themselves with energy and most nutrients by axonal transport. If one places a fibre in a chamber and progressively eliminates nutrients from the surrounding medium, axonal function proceeds normally as long as oxygen is supplied, carbon dioxide is eliminated, and a minimal amount of calcium is present. Glucose and amino acids are not apparently needed since they arrive by axonal transport.

\section{Oxygen levels}

Ernest showed that the oxygen level of the nerve head is constant despite substantial increase in IOP. Either blood flow does not decrease with increased IOP or flow decreases without affecting oxygen delivery. If tissue oxygen tension is increased during acute experimental glaucoma, obstruction of axonal transport is similar to animals breathing room air (an experiment carried out by Minckler in our laboratory). This suggests that oxygen supply at the lamina is unaffected by moderately elevated IOP.

\section{Effects on capillary number}

Christini and François suggested that optic nerve head capillaries are selectively lost in glaucoma. The eyes that they examined were blind and detailed histories were not provided. I studied human and primate glaucoma eyes with quantitative methods. ${ }^{3}$ There is no selective loss of capillaries in the nerve head. Capillaries disappear from the nerve head as nerve fibres disappear, but at just the rate necessary to maintain the usual ratio of capillaries to tissue. This does not prove that blood flow was normal, only that the normal proportion of vessels is unchanged.

\section{Disc haemorrhages}

Capillaries do atrophy in glaucoma, proportionately with loss of neural tissue. What happens to these vessels? Capillaries of the nerve head are a continuous plexus extending from the retina to the myelinated optic nerve. Compression of the lamina cribrosa and loss of surrounding nerve fibre bundles places this plexus under physical stress. Forced to increase in antero-posterior length, and unsupported by adjacent neural tissue, an occasional capillary is stretched to the breaking point and a flame-shaped haemorrhage occurs into the superficial nerve fibre layer of the disc rim. Drance, astutely, noted that such haemorrhages are more likely to occur in glaucomatous eyes that are in the process of developing progressive optic nerve damage. This is certainly compatible with the above explanation. Clinicians must re-evaluate the need for more intense therapy in any glaucomatous eye that has a disc haemorrhage. While there is general agreement about the clinical significance of disc haemorrhage, the pathogenesis of the haemorrhage is not universally agreed upon. It was speculated that disc haemorrhage indicates ischaemia of the nerve head. But there is no direct evidence that haemorrhage results from insufficient vascular function. Haemorrhage occurs with trauma, elevated intravascular pressure, or abnormal capillary fragility. Certainly there are clear differences between the glaucoma eye with a haemorrhage and anterior ischaemic optic neuropathy. The glaucoma patient does not have disc swelling, often does not have an immediately definable new loss of field, and almost never suffers loss of acuity with the event.

\section{Blood flow in human glaucoma: fluorescein angiography}

Unfortunately, there is no reliable method at present to measure human nerve head blood flow in vivo. Fluorescein angiography shows vessels of the superficial nerve head, but capillaries of the scleral lamina are surrounded by collagenous sheets and are not detected in clinical angiography. ${ }^{3}$ Furthermore, fluor- 
escein angiography does not quantitate blood flow. Spaeth demonstrated that glaucomatous discs have a slower filling time than normal discs. While this is interesting, it gives no direct information about the adequacy of nutrition of the nerve head. Slow filling might result if the path from ophthalmic artery to retina is lengthened by cup excavation. Hayreh and Schwartz report that glaucomatous discs have areas without detectable vessels by angiography. Many of the published examples of such 'filling defects' are in eyes with clear nerve loss (large cup/disc ratios and known field defects). As discussed above, at this stage of damage, neuronal loss is accompanied by a proportionate loss of capillaries. Hence, the disc with moderate or severe cup enlargement will naturally have fewer detectable vessels in the anterior disc. Scleral lamina capillaries in the filling defect zones are patent but unseen angiographically. ${ }^{3}$ The fluorescein observations are interesting and understandable in the light of the histological events, but provide no direct evidence for inadequate nutrition to axons.

\section{Blood flow in experimental glaucoma}

In recent experiments, we produced chronic IOP elevation in monkeys by laser treatment of the trabecular meshwork and measured blood flow by a histological method. ${ }^{9}$ In experiments with acute IOP elevations, there was no significant blood flow decrease as long as IOP was kept below $75 \mathrm{~mm} \mathrm{Hg}$. With IOP below $75 \mathrm{~mm} \mathrm{Hg}$, flow was equal to that in normal eyes. In animals with chronic glaucoma, with mean IOP from 25 to $45 \mathrm{~mm} \mathrm{Hg}$, the blood flow in the glaucoma eye was equivalent to normal eyes. Nor was the flow affected to any greater degree in the superior and inferior poles of the nerve head where damage happens most. Sossi and Anderson also found normal flow in cat eyes with acute IOP elevations $(25 \mathrm{~mm} \mathrm{Hg}$ or more below mean blood pressure). Weinstein confirmed that flow was maintained at a stable level in the nerve head despite substantial increases or decreases in systemic blood pressure. This experiment is particularly important to clinicians, since some reports suggested that decrease in brachial blood pressure might decrease blood flow in the optic nerve head. It is clear from Weinstein's work that we should not assume that nerve head flow will change as systemic blood pressure changes. For example, the drug clonidine is not used in the US to lower IOP because it lowers blood pressure. The assumption underlying this reluctance is that the 'perfusion' of the nerve head (blood pressure minus IOP) would not be helped if the drug lowered both. It is clear that drugs can change blood pressure with no detectable effect on blood flow. Sperber and Bill have shown that aerobic metabolism continues normally in the nerve head despite IOP elevation until IOP leaves the clinical range (comes within $25 \mathrm{~mm} \mathrm{Hg}$ of mean blood pressure).

\section{Faulty autoregulation}

Sossi and Anderson speculate that normal autoregulation might not be present in the glaucomatous eye. They suggest that circulating vasoconstrictors might influence nerve head blood flow by entering from the choroid. Weinstein found, however, that a potent vasoconstrictor, metaraminol, substantially affected the systemic vasculature without increasing or decreasing nerve head blood flow. The suggestion that glaucoma damage consists of elevated IOP plus faulty autoregulation overlooks the fact that normal human eyes or experimental monkey eyes that suffer chronic IOP elevation develop glaucomatous optic neuropathy.

\section{Low tension glaucoma}

Some eyes appear to have glaucomatous disc cupping and field loss with apparently normal IOP. Some feel that these eyes support vascular insufficiency as a mechanism of glaucoma damage. The argument is made that if these patients do not have high IOP, then what could have damaged them? The ready conclusion is that they have deficient blood supply-yet there is no direct evidence for this. These eyes might have an optic neuropathy caused by early senescence of neurons or a toxic neuropathy with an as-yet unidentified agent. The most interesting paradox of low tension glaucoma is the fact that they have disc cup configurations similar to "high IOP" glaucomatous eyes. How did the cup come to look this way? Either the lamina became distorted without elevated IOP or IOP was elevated at some point but went 
undetected. If the lamina spontaneously stretched into a glaucomatous shape, then the connective tissues of these eyes might be inherently different. This stretching cannot be produced by experimental vascular insufficiency, so it must be a primary defect. Alternatively, IOP might have been elevated in the past, or is still elevated intermittently, requiring repeated monitoring to detect it.

\section{Cavernous optic nerve degeneration}

Schnabel reported that glaucomatous nerves suffer a cavernous optic atrophy, in which spaces in the retrobulbar nerve are filled with hyaluronic acid. Similar pathology was reported in other optic neuropathies. The similar histologic end-results were interpreted to mean that the pathogenesis of the disorders must be the same. Because optic nerve infarction from giant cell arteritis was among the conditions with cavernous atrophy, it was assumed that this supported infarction as a cause of glaucomatous neuropathy. In 150 glaucoma optic nerves, I found only four with cavernous degeneration. These were from cases with rapid loss of all vision from very high IOP. In chronic, moderate IOP elevation eyes, neurons atrophy without detectable caverns. Why does cavernous degeneration occur? There is a normal movement of fluid from vitreous cavity to retrobulbar nerve tissue. This flow increases if IOP is elevated. With a rapid loss of many nerve fibres, the unoccupied space in the retrobulbar nerve fills with fluid containing vitreous hyaloronate. This happens in any rapid atrophy, regardless of its cause. Acute or neovascular glaucoma would be commonly associated, and these eyes would have come to Schnabel's pathology laboratory, removed as blind and painful. Yet, other disorders share this common pathway, accounting for reported non-glaucomatous cavernous degeneration.

\section{Selective Loss of Particular Ganglion Cell Types}

Our inability to detect early glaucoma damage has been hampered by the lack of knowledge of which retinal ganglion cells are the first to disappear. Recent experimental work in mammals has shown that there are a variety of ganglion cell types. Some cells are small in size and subserve colour vision and fine acuity. Others are large and are colour insensitive, giving instead information on motion or presence/absence of illumination. These cell types have particular terminal zones for their axonal projections in the brain. We have produced chronic experimental glaucoma in monkeys and have traced which ganglion cell types are most susceptible to glaucoma damage. Both in studies of central connections and in measures of cell diameter,,$^{10}$ cells of larger size are more sensitive to damage than are smaller cells. This will direct us to develop psychophysical tests that are geared to the functions that these cells perform.

\section{References}

${ }^{1}$ Quigley HA and Anderson DR: Cupping of the optic disc in ischemic optic neuropathy. Trans. Am. Acad. Ophthalmol. Otolaryngol. 1977, 83: 75562.

${ }^{2}$ Quigley HA, Addicks EM and Green WR: Optic nerve damage in human glaucoma. III. Quantitative correlation of nerve fiber loss and visual field defect in glaucoma, ischemic neuropathy, papilledema, and toxic neuropathy. Arch. Ophthalmol. 1982, 100: 135-46.

${ }^{3}$ Quigley HA, Hohman RM, Addicks EM and Green WR: Blood vessels of the optic disk in chronic glaucoma. Invest. Ophthalmol. Visual Sci. 1984, 25: 918-31.

${ }^{4}$ Quigley HA, Addicks EM, Green WR and Maumenee AE: Optic nerve damage in human glaucoma. II. The site of injury and susceptibility to damage. Arch. Ophthalmol. 1981, 99: 635-49.

5 Vrabec F: Glaucomatous cupping of the human optic disk. A neurohistologic study. Albrecht. von Graefes Archiv. klin. exp. Ophthalmol. 1976, 198: 223-4.

${ }^{6}$ Kirsch RE and Anderson DR: Identification of the glaucomatous disc. Trans. Am. Acad. Ophthalmol. Otolaryngol. 1973, 77: 143-60.

${ }^{7}$ Quigley HA and Addicks EM: Regional differences in the structure of the lamina cribrosa and their relation to glaucomatous optic nerve damage. Arch. Ophthalmol. 1981, 99: 137-43.

${ }^{8}$ Quigley HA, Hohman RM, Addicks EM, Massof RS and Green WR: Morphologic changes in the lamina cribrosa correlated with neural loss in openangle glaucoma. Am. J. Ophthalmol. 1983, 95: 673-91.

${ }^{9}$ Quigley HA, Hohman RM, Sanchez R and Addicks EM: Optic nerve head blood flow in chronic experimental glaucoma. Arch. Ophthalmol. 1985, 103: 956-62.

${ }^{10}$ Quigley HA, Sanchez RM, Dunkelberger GR, L'Hernault NL and Baginski TA: Chronic glaucoma selectively damages large optic nerve fibers. Arch. Ophthalmol. 1986 (submitted for publication). 